\title{
Fine-mapping of qTGW2, a quantitative trait locus for grain weight in rice (Oryza sativa L.)
}

\author{
Hui Zhang ${ }^{1,2,3}$, Yu-Jun Zhu ${ }^{2}$, An-Dong Zhu ${ }^{2}$, Ye-Yang Fan ${ }^{2}$, Ting-Xu Huang ${ }^{3}$, Jian-Fu Zhang ${ }^{3}$, Hua-An Xie ${ }^{\text {Corresp., } 1,3}$ \\ Jie-Yun Zhuang ${ }^{\text {Corresp. } 2}$ \\ ${ }^{1}$ College of Agriculture, Fujian Agriculture and Forestry University, Fuzhou, China \\ 2 State Key Laboratory of Rice Biology and Chinese National Center for Rice Improvement, China National Rice Research Institute, Hangzhou, China \\ 3 Rice Research Institute and Fuzhou Branch of the National Center for Rice Improvement, Fujian Academy of Agricultural Sciences, Fuzhou, China \\ Corresponding Authors: Hua-An Xie, Jie-Yun Zhuang \\ Email address: huaanxie@163.com, zhuangjieyun@caas.cn
}

Background. Grain weight is a grain yield component, which is an integrated index of grain length, width and thickness. They are controlled by a large number of quantitative trait loci (QTLS). Besides major QTLS, minor QTLs play an essential role. In our previously studies, QTL analysis for grain length and width was performed using a recombinant inbred line population derived from rice cross TQ/IRBB lines. Two major QTLs were detected, which were located in proximity to GS3 and G W5 that have been cloned. In the present study, QTLs for grain weight and shape were identified using rice populations that were homozygous at GS3 and GW5. Method. Nine populations derived from the indica rice cross TQ/IRBB52 were used. An $F_{10: 11}$ population named W1, consisting of 250 families and covering 16 segregating regions, was developed from one residual heterozygote $(\mathrm{RH})$ in the $\mathrm{F}_{7}$ generation of Teqing/IRBB52. Three near isogenic line (NIL)- $\mathrm{F}_{2}$ populations, $\mathrm{ZH} 1, \mathrm{ZH} 2$ and ZH3 that comprised 205, 239 and 234 plants, respectively, were derived from three RHs in $F_{10: 11}$. They segregated the target QTL region in an isogenic background. Two NIL populations, HY2 and HY3, were respectively produced from homozygous progeny of the ZH2 and ZH3 populations. Three other NIL-F $F_{2}$ populations, Z1, Z2 and Z3, were established using three RHs having smaller heterozygous segments. QTL analysis for 1000-grain weight (TGW), grain length (GL), grain width (GW), and length/width ratio (LWR) was conducted using QTL IciMapping and SAS procedure with GLM model. Result. A total of 27 QTLs distributed on 12 chromosomes were identified. One QTL cluster, qTGW2/qGL2/qGW2 located in the terminal region of chromosome 2, were selected for further analysis. Two linked QTLs were separated in region Tw31911 - RM266. qGL2 was located in Tw31911-Tw32437 and mainly controlled GL and GW. The effects were larger on GL than on GW and the allelic directions were opposite. qTGW2 was located in Tw35293-RM266 and affected TGW, GL and GW with the same allelic direction. Finally, qTGW2 was 
delimited within a 103-kb region flanked by Tw35293 and Tw35395. Conclusion. qTGW2 with significant effects on TGW, GL and GW was validated and fine-mapped using NIL and NIL- $\mathrm{F}_{2}$ populations. These results provide a basis for map-based cloning of qTGW2 and utilization of $q T G W 2$ in the breeding of high-yielding rice varieties. 
1 Fine-mapping of qTGW2, a quantitative trait locus for grain weight in rice (Oryza

2 sativa L.)

3

4

5

6 Hui Zhang ${ }^{1,2,3}$, Yu-Jun Zhu'², An-Dong Zhu'², Ye-Yang Fan², Ting-Xu Huang ${ }^{3}$, Jian-Fu Zhang ${ }^{3, *}$, Hua-An

7 Xie ${ }^{1,3, *}$, Jie-Yun Zhuang ${ }^{2, *}$

8

$9 \quad{ }^{1}$ College of Agriculture, Fujian Agriculture and Forestry University, Fuzhou 350002, China

$10{ }^{2}$ State Key Laboratory of Rice Biology and Chinese National Center for Rice Improvement, China

11 National Rice Research Institute, Hangzhou 310006, China

12 Rice Research Institute and Fuzhou Branch of the National Center for Rice Improvement, Fujian

13 Academy of Agricultural Sciences, Fuzhou 350018, China

14

15 Corresponding Author:

16 Jie-Yun Zhuang

17359 Tiyuchang Road, Hangzhou, Zhejiang, 310006, China

18 Email address: zhuangjieyun@caas.cn

19 Hua-An Xie

20 Cangshan District, Fuzhou, Fujian, 350018, China

21 Email address: huaanxie@163.com

22 Jian-Fu Zhang

23 Cangshan District, Fuzhou, Fujian, 350018, China

24 Email address: jianfzhang@163.com 


\section{Abstract}

26 Background. Grain weight is a grain yield component, which is an integrated index of grain length, width and thickness. They are controlled by a large number of quantitative trait loci (QTLs). Besides major QTLs, minor QTLs play an essential role. In our previously studies, QTL analysis for grain length and width was performed using a recombinant inbred line population derived from rice cross TQ/IRBB lines. Two major QTLs were detected, which were located in proximity to GS3 and GW5 that have been cloned. In the present study, QTLs for grain weight and shape were identified using rice populations that were homozygous at GS3 and GW5. Method. Nine populations derived from the indica rice cross TQ/IRBB52 were used. An F10:11 population named W1, consisting of 250 families and covering 16 segregating regions, was developed from one residual heterozygote $(\mathrm{RH})$ in the $\mathrm{F}_{7}$ generation of Teqing/IRBB52. Three near isogenic line (NIL)-F F $_{2}$ populations, ZH1, ZH2 and ZH3 that comprised 205, 239 and 234 plants, respectively, were derived from three RHs in $\mathrm{F}_{10: 11}$. They segregated the target QTL region in an isogenic background. Two NIL populations, HY2 and HY3, were respectively produced from homozygous progeny of the ZH2 and ZH3 populations. Three other NIL-F populations, Z1, Z2 and Z3, were established using three RHs having smaller heterozygous segments. QTL analysis for 1000-grain weight (TGW), grain length (GL), grain width (GW), and length/width ratio (LWR) was conducted using QTL IciMapping and SAS procedure with GLM model.

Result. A total of 27 QTLs distributed on 12 chromosomes were identified. One QTL cluster, qTGW2/qGL2/qGW2 located in the terminal region of chromosome 2, were selected for further analysis. Two linked QTLs were separated in region Tw31911-RM266. qGL2 was located in Tw31911-Tw32437 and mainly controlled GL and GW. The effects were larger on GL than on GW and the allelic directions were opposite. qTGW2 was located in Tw35293-RM266 and affected TGW, GL and GW with the same allelic direction. Finally, qTGW2 was delimited within a 103-kb region flanked by Tw35293 and Tw35395.

Conclusion. qTGW2 with significant effects on TGW, GL and GW was validated and finemapped using NIL and NIL-F 2 populations. These results provide a basis for map-based cloning of $q T G W 2$ and utilization of $q T G W 2$ in the breeding of high-yielding rice varieties.

54 Minor effect; Rice

\section{Introduction}


57 Rice is one of the staple food crops and consumed by half of the world's population. Enhancing

58 grain yield is always among main objectives of breeding programs. Grain weight is a key

59 component of grain yield in rice, which is mainly determined by grain length, width and

60 thickness. All these traits are quantitatively inherited and controlled by both major and minor

61 genes.

More than 500 quantitative trait loci (QTLs) for grain weight and shape have been identified previously (http://www.gramene.org). So far, 16 QTLs for these traits with large effects were cloned using diverse mapping populations. Among these, ten genes regulate grain weight by mainly affected grain length, including GS2/GL2, OsLG3, OsLG3b, GS3, GL3.1/qGL3, qTGW3/ TGW3/GL3.3, TGW6, GW6a, GL6, and GLW7 (Fan et al., 2006; Qi et al., 2012; Zhang et al., 2012; Ishimaru et al., 2013; Hu et al., 2015; Song et al., 2015; Che et al., 2016; Si et al. 2016; Yu et al., 2017; Hu et al., 2018; Xia et al., 2018; Ying et al., 2018; Yu et al., 2018; Wang et al., 2019). Other four genes regulate grain weight by primarily influencing grain width, including GW2, GS5, GSE5 and GW8 (Song et al., 2007; Li et al., 2011; Wang et al., 2012; Duan et al., 2017). The remaining two genes, GL7/GW7, and GS9, have similar influences on grain length and width with opposite allelic directions, thus they hardly affect grain weight (Wang et al., 2015b; Wang et al., 2015c; Zhao et al., 2018). Identification of these genes have facilitated the breeding of high-yield rice varieties.

Although it has been recognized that both major and minor QTLs play essential roles in the genetic control of complex traits (Mackay, Stone \& Ayroles 2009), identification of minor QTLs has been limited due to their small effects across different genetic backgrounds and environments. Recently, more and more studies have paid attention to minor QTLs. A number of minor QTLs for grain weight and shape were fine-mapped, such as qTGW1.1a, qTGW1.2a, qGS1-35.2, qGW1-35.5, and qTGW10-20.8 (Zhang et al., 2016; Dong et al., 2018; Wang et al., 2019; Zhu et al., 2019b). Isolation of more minor QTLs would be beneficial for establishing the network regulating grain weight in rice.

In our previous studies, QTL analysis for grain length and width was performed using recombinant inbred lines (RILs) of indica rice crosses between Teqing (TQ) and IRBB lines. Two major QTLs were detected, which were located in approximate to GS3 and GW5, respectively (Wang et al., 2017). The present study aims to identify QTLs for grain weight and shape after eliminating the segregation of GS3 and GW5. Firstly, QTLs for grain weight and shape were detected using an $\mathrm{F}_{10: 11}$ population that was derived from one residual heterozygote 
90 was selected for validation, dissection and fine-mapping. Using two sets of near isogenic lines

91 (NIL) and six NIL-F 2 populations derived from the $\mathrm{F}_{10: 11}$ population, two QTLs were separated in 92 the target region. One of them, qTGW2, was delimited into a 103-kb region flanked by Tw35293 93 and Tw35395.

\section{Materials \& Methods}

95 Plant materials

96 Nine mapping populations of indica rice were used in this study. The first one was an $\mathrm{F}_{10: 11}$

97 population that was previously developed from one $\mathrm{RH}$ in the $\mathrm{F}_{7}$ generation of TQ/IRBB52

98 (Zhang et al., 2019), consisting of 250 families and segregated 16 regions distributed on the 12

99 rice chromosomes. This population was named W1. The remaining eight populations were

100 developed from RHs selected from the W1 population as described below and illustrated in Fig.

101 1. Three plants in $F_{10}$, which carried heterozygous segments that covered partial or entire region

102 of the interval Tw31911-RM266 on chromosome 2, were identified. They were selfed to produce

103 three NIL-F 2 populations consisting of 205, 239 and 234 plants and named ZH1, ZH2 and ZH3,

104 respectively. Two QTLs were separated, of which qTGW2 located in the downstream region was

105 selected for further analysis. Non-recombinant homozygotes were identified in the ZH2 and ZH3

106 populations and selfed. Two sets of NILs named HY2 and HY3 were developed, each consisting

107 of 35 TQ homozygous lines and 35 IRBB52 homozygous lines. They were used to validate

108 qTGW2. Then, other three RHs, carrying heterozygous segments overlapped in the terminal

109 region Tw35293-RM266 of chromosome 2, were identified from the W1 population. The three

110 plants were selfed to produce three NIL-F $F_{2}$ populations in $F_{12}$, which consisted of 174, 237 and

111228 plants and named Z1, Z2 and Z3, respectively.

\section{Field experiment and trait measurement}

113 The rice populations were planted in the experimental stations of the China National Rice

114 Research Institute located at either Lingshui in Hainan Province or Hangzhou in Zhejiang

115 Province. For the $F_{10: 11}$ population and two sets of NILs, the experiments followed a randomized

116 complete block design with two replications. For each replication, twelve plants per line were

117 planted in one row. At maturity, five of the middle ten plants in each row were harvested in bulk

118 and sun-dried. Two samples of approximately $10 \mathrm{~g}$ fully filled grains were randomly selected for

119 the measurement of TGW, GL, GW, and length/width ratio (LWR) following the procedure

120 reported by Zhang et al. (2016). For the six NIL-F 2 populations, plants were harvested 
121 individually and sun-dried. Two samples of approximately $3 \mathrm{~g}$ fully filled grains of each plant

122 were selected for the measurement of the four traits.

\section{DNA marker analysis}

124 For the W1 population and two NIL populations, leaf samples collected from the middle eight 125 plants of a rice line were mixed for DNA extraction using a mini-preparation protocol (Zheng et 126 al., 1995). For the six NIL-F $F_{2}$ populations, a $2 \mathrm{~cm}$-long leaf sample collected from an $\mathrm{F}_{2}$ plant was 127 used for DNA extraction using the same method. PCR amplification was performed according to 128 Chen et al. (1997). The products were visualized on 6\% non-denaturing polyacrylamide gels 129 using silver staining. A total of 68 polymorphic markers were used, including 57 simple sequence 130 repeats, eight insertion/deletions, one cleaved amplified polymorphic sequence, and two sequence 131 tagged sites. Nine of them were developed according to sequence differences between TQ and 132 IRBB52 detected with whole-genome resequencing (Table S1).

\section{Data analysis}

134 For the W1 and six NIL-F2 populations, genetic maps of each populations were constructed using 135 Mapmaker/Exp 3.0, in which genetic distances between markers were presented in centiMorgan 136 (cM) derived with Kosambi function. QTL mapping was performed using the default setting of 137 the BIP (QTL mapping in bi-parental populations) approach in IciMapping V4.1 (Meng et al., 138 2015). LOD thresholds were calculated with 1000 permutation test $(P<0.05)$ and used to claim a 139 putative QTL.

140 For the two NIL populations, two-way analysis of variance (ANOVA) was performed to test

141 the phenotypic differences between the two genotypic groups in each NIL set. The analysis was 142 performed using the SAS procedure GLM (SAS Institute, 1999) as described previously (Dai et 143 al., 2008). Given the detection of a significant difference $(P<0.05)$, the same data were used to 144 estimate the genetic effect of the QTL, including additive effect and the proportion of phenotypic 145 variance explained $\left(R^{2}\right)$. QTL were designated according to the rules recommended by McCouch 146 and CGSNL (2008).

\section{Results}

\section{QTLs detected in the W1 population}

149 A total of 27 QTLs for the four traits were detected, which were distributed on 14 segregating 150 regions (Fig. 2, Table 1). Four of them had significant effects on three traits. In the 151 Tw35293-RM266 region on chromosome 2, the IRBB52 allele increased TGW, GL and GW by $1520.39 \mathrm{~g}, 0.060 \mathrm{~mm}$ and $0.013 \mathrm{~mm}$, respectively. In the RM14032-RM14383 interval on 
153 chromosome 3, the IRBB52 allele increased GL by $0.025 \mathrm{~mm}$, decreased GW by $0.014 \mathrm{~mm}$ and

154 increased LWR 0.022. In the RM16252-RM335 region on chromosome 4, the IRBB52 allele

155 decreased TGW and GW by $0.18 \mathrm{~g}$ and $0.017 \mathrm{~mm}$, respectively, but increased LWR by 0.021 . In

156 the Tv963-RM27610 interval on chromosome 12, the IRBB52 allele increased GL by 0.031mm,

157 decreased GW by $0.013 \mathrm{~mm}$ and increased LWR 0.027. Worthy to note, the Tw35293-RM266

158 region had $R^{2}$ of $14.50 \%$ for TGW and $18.60 \%$ for GL, which were much higher than the $R^{2}$

159 values for these two traits detected in the other three regions.

160 Five regions had significant effects on two traits. In the RM12210 region on chromosome 1

161 the IRBB52 allele increased GL and LWR by $0.026 \mathrm{~mm}$ and 0.011 , respectively. In the

162 RM3321-RM274 interval on chromosome 5, the IRBB52 allele increased GL and LWR by 0.062

$163 \mathrm{~mm}$ and 0.018, respectively. In the RM549 region on chromosome 6, the IRBB52 allele increased

164 TGW and GW by $0.27 \mathrm{~g}$ and $0.012 \mathrm{~mm}$, respectively. In the interval RM22755-RM23001 on

165 chromosome 8, the IRBB52 allele reduced GW by $0.009 \mathrm{~mm}$ and increased LWR by 0.017 . In

166 the interval RM1108-RM7300 on chromosome 10, the IRBB52 allele reduced GW by $0.004 \mathrm{~mm}$

167 and increased LWR by 0.012 .

168 The remaining five QTLs were unaccompanied with other QTLs, including qTGW7 for

169 TGW, qGW9.1 and $q G W 9.2$ for GW, and $q L W R 11$ and $q L W R 12.2$ for LWR.

170 Dissection of two QTLs for grain size on chromosome 2

171 As described above, the terminal region of chromosome 2 had relatively large effects in terms of

172 the number of QTLs detected and $R^{2}$ of single QTL. Therefore, this region was chosen for further

173 validation and fine-mapping.

174 Three NIL-F 2 populations, ZH1, ZH2 and ZH3, were constructed, following the results of the

175 W1 population (Fig. 3A). To fill the long distance between Tw32437 and Tw35293, two

176 polymorphic markers, RM14034 and RM14056, were selected (Table S1). The two markers were

177 homozygous in all the three populations. Two segregating regions, Tw31911-Tw32437 and

178 Tw35293-RM266, were separated in the Z1 population (Fig. 3B). As shown in Table 2, QTLs

179 were detected in both regions. In the interval Tw31911-Tw32437, the IRBB52 allele decreased

180 TGW and GL but increased GW and LWR, having $R^{2}$ of 6.05, 29.51, 13.61 and 28.52 \%,

181 respectively. QTLs in this region affected GL and GW with opposite directions. The effect was

182 larger on GL than on GW, resulting in the detection of a residual effect on TGW. Thus, this QTL

183 was nominated as qGL2. In the Tw35293-RM266 region, the IRBB52 allele increased TGW, GL

184 and GW, having $R^{2}$ of 31.76, 17.95 and $3.73 \%$, respectively. QTLs in this region affected GL 
185 and GW with the same direction, and the accumulative effect resulted in larger influence on

186 TGW. Thus, this QTL was nominated as qTGW2.

187 The ZH2 and ZH3 populations were only segregated in the Tw35293-RM266 region. In

188 both populations, significant effects were detected on all the traits except LWR. The enhancing

189 alleles were always derived from IRBB52, and the effects were similar between the two

190 populations. The additive effects were 0.55 and $0.46 \mathrm{~g}$ on TGW, 0.065 and $0.061 \mathrm{~mm}$ on GL, and

1910.021 and $0.020 \mathrm{~mm}$ on GW. The $R^{2}$ were 28.63 and $21.83 \%$ for TGW, 22.34 and $28.86 \%$ for

192 GL, and 22.91 and 22.37 \% for GW. These results are similar to those found in the ZH1

193 population, indicating that qTGW2 located in the interval Tw35293-RM266 affects TGW, GL

194 and GW with the same allelic direction. Since qTGW2 showed stable effects across the three

195 populations, this QTL was selected for further analysis.

196 Validation and fine-mapping of qTGW2

197 Two sets of NILs, HY2 and HY3, were used to validate the genetic effects of qTGW2. Frequency

198 distributions of the four traits were plotted using the two genotypic groups as two series (Fig. 4).

199 For TGW, GL and GW, the difference between the TQ and IRBB52 homozygous genotypes were

200 observed in both the populations. The IRBB52 homozygous lines were distributed in the higher-

201 value region, and the TQ homozygous lines were distributed in the lower-value region. On the

202 other hand, no distinction was found for LWR. These results indicate that QTLs for TGW, GL

203 and GW were segregated in the two populations with the enhancing alleles derived from IRBB52.

204 Results of the two-way ANOVA on the four traits are presented in Table 3. Highly

205 significant effects $(P<0.0001)$ were detected for TGW, GL and GW in both the HY2 and HY3

206 populations. The effects were similar between the two populations, with the IRBB52 allele

207 always increasing the trait values. The additive effects were 0.47 and $0.45 \mathrm{~g}$ on TGW, 0.054 and

$2080.061 \mathrm{~mm}$ on GL, and 0.021 and $0.013 \mathrm{~mm}$ on GW. The $R^{2}$ were 60.94 and $66.02 \%$ for TGW,

20948.26 and $62.12 \%$ for GL, and 36.67 and $22.74 \%$ for GW. In addition, significant influence on

210 LWR were only detected in the HY3 population $(P=0.0003)$. The IRBB52 allele increased LWR

211 by 0.009 with the $R^{2}$ of $12.94 \%$. It was found that the allelic direction of qTGW2 remained

212 unchanged across the five populations, with the IRBB52 allele always increasing TGW, GL and

213 GW. As compared to the ZH1, ZH2 and ZH3 populations, the additive effect of qTGW2 hardly

214 changed but the $R^{2}$ values increased greatly in HY2 and HY3.

215 To further narrow down the region of $q T G W 2$, three polymorphic markers, RM14189,

216 Tw35277 and Tw35395, were added (Table S1). Three plants were identified from the W1 
217 population and selfed to produce three NIL-F2 populations named Z1, Z2 and Z3. As shown in

218 Figure 3C, the segregating regions in Z1, Z2 and Z3 were RM14189-Tw35293,

219 Tw35293-RM266, and Tw35395-RM266, respectively. QTL analysis for TGW, GL, GW and

220 LWR were conducted (Table 4). Significant effects were detected in Z2 but not in the other two

221 populations. This result suggests that qTGW2 was segregated in Z2 but not in Z1 and Z3.

222 Consequently, the qTGW2 was delimited within a 103-kb region flanked by Tw35293 and

223 Tw35395. In Z2, the IRBB52 allele increased TGW by $0.46 \mathrm{~g}$, GL by $0.053 \mathrm{~mm}$ and GW by

$2240.014 \mathrm{~mm}$, with the $R^{2}$ of 29.93, 16.26 and $13.70 \%$, respectively.

\section{Discussion}

226 Although progress has been made in fine mapping and cloning of major genes for grain weight,

227 experimental constraints have limited our knowledge of minor genes that could be responsible for

228 a larger proportion of trait variation. In this study, 27 QTLs for grain weight and shape in rice

229 were detected using one population derived from an RH that was homozygous at major QTLs

230 detected previously, followed by delimitation of qTGW2 for grain weight, length and width into a

231 103-kb region on chromosome 2.

232 All the populations used in this study were constructed from a single $\mathrm{F}_{7}$ plant of an indica

233 rice cross. Among them, three NIL-F 2 populations in $\mathrm{F}_{11}$ were grown under short-day conditions

234 in Lingshui, and others were grown under long-day conditions in Hangzhou. qTGW2 showed

235 stable effects on TGW, GL and GW across these populations. The IRBB52 allele increased

$236 \mathrm{TGW}, \mathrm{GL}$ and GW by a range of 0.45 to $0.55 \mathrm{~g}, 0.053$ to $0.065 \mathrm{~mm}$, and 0.006 to $0.021 \mathrm{~mm}$,

237 respectively. These results support that minor QTLs could be steadily detected in a highly

238 isogenic background despite of diverse environment conditions, and the use of RHs could be an

239 efficient way to detect and fine map minor QTLs.

240 Among the 16 QTLs cloned for grain weight and shape with major effects, GS3, OsLG3,

241 OsLG3b, GS5, GSE5, GW6a, GL7/GW7, GLW7 and GW8 were found with high frequency in the

242 modern rice varieties (Yan et al., 2009; Wang, Chen \& Yu, 2011; Takano et al., 2009; Mao et al.,

243 2010; Yu et al., 2017; Yu et al., 2018; Li et al., 2011; Duan et al., 2017; Wang et al., 2015; Si et

244 al., 2016). Two of the other QTLs, GW2 and GS2/GL2/GLW2 on chromosome 2 were rarely

245 found in modern rice varieties. qTGW2 identified in this study was located in the interval

246 Tw35293-Tw35395, corresponding to the 35.3-35.4 Mb region on the terminal end of

247 chromosome 2, which was 6.4 Mb away from GS2 locus in the Nipponbare genome (IRGSP,

248 2005). The interval RM6-RM240 covering GS2 was detected as a non-segregating region in the

249 populations used in the present study (Fig. 2). These results suggest that qTGW2 identified in 
250 this study is likely a new QTL for grain weight. Cloning and functional characterization of

251 qTGW2 would provide new information for understanding the genetic and molecular basis of 252 grain weight in rice.

253 Based on the Rice Genome Annotation Project (http://rice.plantbiology.msu.edu), there are 25416 annotated genes in the 103-kb region for qTGW2 (Table S2). Thirteen of these genes encode 255 known proteins, among which LOC_Os02g57630 encodes ubiquitin carboxyl-terminal hydrolase, 256 LOC_Os02g57640 encodes a protein with the KH domain, LOC_Os02g57650 encodes a no 257 apical meristem protein, LOC_Os02g57660 encodes phosphatidylinositol-4-phosphate 5-kinase, 258 LOC_Os02g57670 encodes ribosomal L9, LOC_Os02g57690 encodes a kelch repeat protein, 259 LOC_Os02g57700 encodes protein kinase, LOC_Os02g57710 encodes signal peptide peptidase260 like 2B, LOC_Os02g57750 encodes a protein binding protein, LOC_Os02g57760 encodes O261 methyltransferase, LOC_Os02g57770 encodes glycosyl hydrolases family 16, LOC_Os02g57790 262 encodes a ZOS2-19-C2H2 zinc finger protein, and LOC_Os02g57720 encodes an aquaporin 263 protein. LOC_Os02g57720 may correspond to RWC3 and OsPIP2a. RWC3 was involved in the 264 regulation of rice drought avoidance (Lian et al., 2004), and the expression of OsPIP2a in rapidly 265 growing internodes of rice is not primarily controlled by meristem activity or cell expansion 266 (Malz \& Sauter, 1999). Of the remaining three annotated genes, LOC_Os02g57740 and 267 LOC_Os02g57780 encode uncharacterized expressed proteins, and LOC_Os02g57730 encodes 268 hypothetical protein. Further analyses are needed to confirm the candidate gene for qTGW2. 269 In addition to the qGL2- qTGW2 cluster on the terminal end of chromosome 2, a few other regions were detected to have important effects on grain weight and shape in the W1 population (Table 1). One of them, the RM1108-RM7300 region on the long arm of chromosome 10, has been targeted for more studies. Three QTLs were dissected (Zhu et al., 2019a), one of which was delimitated within a 70.7-kb region containing seven annotated genes (Zhu et al., 2019b). Five other regions, RM14302-RM14383 on chromosome 3, RM3321-RM274 on chromosome 5, RM10-RM70 on chromosome 7, RM167-RM287 on chromosome 11 and Tv963-RM27610 on chromosome 12, were previously reported to influence heading date differences between TQ and IRBB52 (Sun et al., 2018). Work is underway to determine the roles of these QTLs on multiple traits in rice.

280 A minor-effect QTL for grain weight, length and width in rice, qTGW2 located in the terminal 281 region on the long arm of chromosome 2, was delimited to a 103-kb region flanked by Tw35293 
282 and Tw35395 using NILs and NIL-F 2 populations. This QTL had a consistent effect across

283 different environment, providing a potential candidate gene for map-based cloning.

284

285 Acknowledgements

286 Funding

287 This work was supported by the Science and Technology Innovation Program of the Fujian

288 Academy of Agricultural Sciences (STIT 2017-1-1), the National Research and Development

289 Program (2016YFD0101801), the Special Foundation of Non-Profit Research Institutes of Fujian

290 Province (2018R1101013-4) and the National Natural Science Foundation of China (31521064).

291 Grant Disclosures

292 The following grant information was disclosed by the authors:

293 Science and Technology Innovation Program of the Fujian Academy of Agricultural Sciences:

294 STIT 2017-1-1.

295 National Research and Development Program: 2016YFD0101801.

296 Special Foundation of Non-Profit Research Institutes of Fujian Province: 2018R1101013-4.

297 National Natural Science Foundation of China: 31521064.

298 Competing of Interests

299 The authors declare that they have no conflict of interests.

300 Author Contributions

301 JYZ, HAX and JFZ conceived the study. HZ and JYZ wrote the manuscript.

$302 \mathrm{HZ}, \mathrm{JYZ}$ and YJZ performed the data analysis.

303 HZ, YJZ, ADZ, YYF and TXH carried out the marker genotyping and the trait phenotyping.

304 All authors read and approved the final manuscript.

305 Data Availability

306 The following information was supplied regarding data availability:

307 The raw data were provided in the Supplemental Table 1 and 2.

308

309

\section{References}


310 Chen X, Temnykh S, XuY, Cho YG, McCouch SR. 1997. Development of a microsatellite

311 framework map providing genome-wide coverage in rice (Oryza sativa L.). Theoretical \&

312 Applied Genetics 95: 553-567 DOI 10.1007/s001220050596.

313 Che R, Tong H, Shi B, Liu Y, Fang S, Liu D, Xiao Y, Hu B, Liu L, Wang H, Zhao M, Chu

314 C. 2016. Control of grain size and rice yield by GL2-mediated brassinosteroid responses.

$315 \quad$ Nature Plants 2: 1-7 DOI 10.1038/NPLANTS.2015.195.

316 Dai W-M, Zhang K-Q, Wu J-R, Wang L, Duan B-W, Zheng K-L, Cai R, Zhuang J-Y. 2008.

317 Validating a segment on the short arm of chromosome 6 responsible for genetic variation in the

318 hull silicon content and yield traits of rice. Euphytica 160: 317-324 DOI 10.1007/s10681-007-

319 9501-8.

320 Dong Q, Zhang Z-H, Wang L-L, Zhu Y-J, Fan Y-Y, Mou T-M, Ma L-Y, Zhuang J-Y. 2018.

321 Dissection and fine-mapping of two QTL for grain size linked in a 460-kb region on

322 chromosome 1 of rice. Rice 11: 44 DOI 10.1186/s12284-018-0236-z.

323 Duan P, Xu J, Zeng D, Zhang B, Geng M, Zhang G, Huang K, Huang L, Xu R, Ge S, Qian

324 Q, Li Y. 2017. Natural variation in the promoter of GSE5 contributes to grain size diversity in rice. Molecular Plant 10: 685-694 DOI 10.1016/j.molp.2017.03.009.

Fan C, Xing Y, Mao H, Lu T, Han B, Xu C, Li X, Zhang Q. 2006. GS3, a major QTL for grain length and weight and minor QTL for grain width and thickness in rice, encodes a putative transmembrane protein. Theoretical \& Applied Genetics 112: 1164-1171 DOI 10.1007/s00122-006-0218-1.

Hu J, Wang Y, Fang Y, Zeng L, Xu J, Yu H, Shi Z, Pan J, Zhang D, Kang S, Zhu L, Dong G, Guo L, Zeng D, Zhang G, Xie L, Xiong G, Li J, Qian Q. 2015. A rare allele of GS2 enhances grain size and grain yield in rice. Molecular Plant 8: 1455-1465 DOI 10.1016/j.molp.2015.07.002.

Hu Z, Lu S-J, Wang M-J, He H, Sun L, Wang H, Liu X-H, Jiang L, Sun J-L, Xin X, Kong W, Chu C, Xue H-W, Yang J, Luo X, Liu J-X. 2018. A novel QTL qTGW3 encodes the GSK3/ SHAGGY-Like Kinase OsGSK5/OsSK41 that Interacts with OsARF4 to negatively regulate grain size and weight in rice. Molecular Plant 11: 736-749 DOI 10.1016/j.molp.2018.03.005.

Ishimaru K, Hirotsu N, Madoka Y, Murakami N, Hara N, Onodera H, Kashiwagi T, Ujiie 342 K, Shimizu B, Onishi A, Miyagawa H, Katoh E. 2013. Loss of function of the IAA-glucose hydrolase gene TGW6 enhances rice grain weight and increase yield. Nature Genetics 45: 707713 DOI 10.1038/ng.2612. 
343

344

345

346

347

348

349

350

351

352

353

354

355

356

357

358

359

360

361

362

363

364

365

366

367

368

369

370

371

372

373

374

Li Y, Fan C, Xing Y, Jiang Y, Luo L, Sun L, Shao D, Xu C, Li X, Xiao J, He Y, Zhang Q.

2011. Natural variation in GS5 plays an important role in regulating grain size and yield in rice. Nature Genetics 43: 1266-1269 DOI 10.1038/ng.977.

Lian H-L, Yu X, Ye Q, Ding X-S, Kitagawa Y, Kwak S-S, Su W-A, Tang Z-C. 2004. The role of aquaporin RWC3 in drought avoidance in rice. Plant Cell Physiology 45: 481-489 DOI 10.1093/pcp/pch058.

Mackay TFC, Stone EA, Ayroles JF. 2009. The genetics of quantitative traits: challenges and prospects. Nature Reviews Genetics 10: 565-577 DOI 10.1038/nrg2612.

Malz S, Sauter M. 1999. Expression of two PIP genes in rapidly growing internodes of rice is not primarily controlled by meristem activity or cell expansion. Plant Molecular Biology 40: 985-995 DOI 10.1023/A:1006265528015.

Mao H, Sun S, Yao J, Wang C, Yu S, Xu C, Li X, Zhang Q. 2010. Linking differential domain functions of the GS3 protein to natural variation of grain size in rice. Proceedings of the National Academy of Sciences of the United States of America 107: 19579-19584 DOI 10.1073/pnas.1014419107.

McCouch SR, CGSNL (Committee on Gene Symbolization, Nomenclature and Linkage, rice genetics cooperative). 2008. Gene nomenclature system for rice. Rice 1: 72-84 DOI 10.1007/s12284-008-9004-9.

Meng L, Li H, Zhang L, Wang J. 2015. QTL IciMapping: Integrated software for genetic linkage map construction and quantitative trait locus mapping in biparental populations. The Crop Journal 3: 269-283 DOI 10.1016/j.cj.2015.01.001.

Qi P, Lin YS, Song XJ, Shen JB, Huang W, Shan JX, Zhu MZ, Jiang L, Gao JP, Lin HX. 2012. The novel quantitative trait locus GL3.1 controls rice grain size and yield by regulating Cyclin-T1:3. Cell Research 22: 1666-1680 DOI 10.1038/cr.2012.151.

SAS Institute Inc. 1999. SAS/STAT User’s Guide. Cary: SAS Institute.

Si L, Chen J, Huang X, Gong H, Luo J, Hou Q, Zhou T, Lu T, Zhu J, Shangguan Y, Chen E, Gong C, Zhao Q, Jing Y, Zhao Y, Li Y, Cui L, Fan D, Lu Y, Weng Q, Wang Y, Zhan Q, Liu K, Wei X, An K, An G, Han B. 2016. OsSPL13 controls grain size in cultivated rice. Nature Genetics 48: 447-456 DOI 10.1038/ng.3518.

Song XJ, Huang W, Shi M, Zhu MZ, Lin HX. 2007. A QTL for rice grain width and weight encodes a previously unknown RING-type E3 ubiquitin ligase. Nature Genetics 39: 623-630 DOI 10.1038/ng2014. 
375 Song XJ, Kuroha T, Ayano M, Furuta T, Nagai K, Komeda N, Segami S, Miura K, Ogawa

376 D, Kmura T, Suzuki T, Higashiyama T, Yamasaki M, Mori H, Inukai Y, Wu J, Kitano H,

377 Sakakibara H, Jacobsen SE, Ashikari M. 2015. Rare allele of a previously unidentified

378 histone $\mathrm{H} 4$ acetyltransferase enhances grain weight, yield, and plant biomass in rice.

379 Proceedings of the National Academy of Sciences of the United States of America 112: 76-81

380 DOI 10.1073/pnas.1421127112.

381 Sun Z, Zhu Y, Chen J, Zhang H, Zhang Z, Niu X, Fan Y, Zhuang J. 2018. Minor-effect QTL

382 for heading date detected in crosses between indica rice cultivar Teqing and near isogenic lines

383 of IR24. The Crop Journal 6: 291-298 DOI 10.1016/j.cj.2018.01.002.

384 Takano-Kai N, Jiang H, Kubo T, Sweeney M, Matsumoto T, Kanamori H,

385 Padukasahasram B, Bustamante C, Yoshimura A, Doi K, McCouch S. 2009. Evolutionary

386 history of GS3, a gene conferring grain length in rice. Genetics 182: 1323-1334 DOI 10.1534/

387 genetics.109.103002.

388 Wang A, Hou Q, Si L, Huang X, Luo J, Lu D, Zhu J, Shangguan Y, Miao J, Xie Y, Wang Y, 389 Zhao Q, Feng Q, Zhou C, Li Y, Fan D, Lu Y, Tian Q, Wang Z, Han B. 2019. The PLATZ

390 transcription factor GL6 affects grain length and number in rice. Plant Physiology 180:

391 2077-2090 DOI 10.1104/pp.18.01574.

392 Wang L-L, Chen Y-Y, Guo L, Zhang H-W, Fan Y-Y, Zhuang J-Y. 2015a. Dissection of

393 qTGW1.2 to three QTLs for grain weight and grain size in rice (Oryza sativa L.). Euphytica

394 202: 119-127 DOI 10.1007/s10681-014-1237-7.

395

Wang C, Chen S, Yu S. 2011. Functional markers developed from multiple loci in GS3 for fine markers-assisted selection of grain length in rice. Theoretical \& Applied Genetics 122: 905913 DOI 10.1007/s00122-010-1497-0.

Wang S, Li S, Liu Q, Wu K, Zhang J, Wang S, Wang Y, Chen X, Zhang Y, Gao C, Wang F, Huang H, Fu X. 2015b. The OsSPL16-GW7 regulatory module determines grain shape and simultaneously improves rice yield and grain quality. Nature Genetics 47: 949-954 DOI 10.1038/ng.3352.

Wang S, Wu K, Yuan Q, Liu X, Liu Z, Lin X, Zeng R, Zhu H, Dong G, Qian Q, Zhang G, Fu X. 2012. Control of grain size, shape and quality by OsSPL16 in rice. Nature Genetics 44: 950-955 DOI 10.1038/ng.2327.

Wang W, Wang L, Zhu Y, Fan Y, Zhuang J. 2019. Fine-mapping of $q T G W 1.2 a$, a quantitative 407 trait locus for 1000-grain weight in rice (Oryza sativa L.). Rice Science 26: 220-228 DOI 10.1016/j.rsci.2019.04.004. 
408

409

410

411

412

413

414

415

416

417

418

419

420

421

422

423

424

425

426

427

428

429

430

431

432

433

434

435

436

437

438

Wang Y, Xiong G, Hu J, Jiang L, Yu H, Xu J, Fang Y, Zeng L, Xu E, Xu J, Ye W, Meng X, Liu R, Chen H, Jing Y, Wang Y, Zhu X, Li J, Qian Q. 2015c. Copy number variation at the GL7 locus contributes to grain size diversity in rice. Nature Genetics 47: 944-948 DOI 10.1038/ng.3346.

Wang Z, Chen J-Y, Zhu Y-J, Fan Y-Y, Zhuang J-Y. 2017. Validation of qGS10, a quantitative trait locus for grain size on the long arm of chromosome 10 in rice (Oryza sativa L.). Journal of Integrative Agriculture 16: 20-30 DOI 10.1016/S2095-3119(16)61410-7.

Wu W, Liu X, Wang M, Meyer RS, Luo X, Ndjiondjop M-N, Tan L, Zhang J, Wu J, Cai H, Sun C, Wang X, Wing RA, Zhu Z. 2017. A single-nucleotide polymorphism causes smaller grain size and loss of seed shattering during African rice domestication. Nature Plants 3: 17064 DOI 10.1038/nplants.2017.64.

Xia D, Zhou H, Liu R, Dan W, Li P, Wu B, Chen J, Wang L, Gao G, Zhang Q, He Y. 2018. GL3.3, a novel QTL encoding a GSK3/SHAGGY-like kinase, epistatically interacts with GS3 to form extra-long grains in rice. Molecular Plant 11: 754-756 DOI 10.1016/j.molp.2018.03.006.

Yan C-J, Yan S, Yang Y-C, Zeng X-H, Fang Y-W, Zeng S-Y, Tian C-Y, Sun Y-W, Tang SZ, Gu M-H. 2009. Development of gene-tagged markers for quantitative trait loci underlying rice yield components. Euphytica 169: 215-226 DOI 10.1007/s10681-009-9937-0.

Ying J-Z, Ma M, Bai C, Huang X-H, Liu J-L, Fang Y-Y, Song X-J. 2018. TGW3, a major QTL that negatively modulates grain length and weight in rice. Molecular Plant 11: 750-753 DOI 10.1016/j.molp.2018.03.007.

Yu J, Miao J, Zhang Z, Xiong H, Zhu X, Sun X, Pan Y, Liang Y, Zhang Q, Rashid MAR, Li J, Zhang H, Li Z. 2018. Alternative splicing of OsLG3b controls grain length and yield in japonica rice. Plant Biotechnology Journal 16: 1667-1678 DOI 10.1111/pbi.12903.

Yu J, Xiong H, Zhu X, Zhang H, Li H, Miao J, Wang W, Tang Z, Zhang Z, Yao G, Zhang Q, Pan Y, Wang X, Rashid M A R, Li J, Gao Y, Li Z, Yang W, Fu X, Li Z. 2017. OsLG3 contributing to rice grain length and yield was mined by Ho-LAMap. BMC Biology 15: 28 DOI 10.1186/s12915-017-0365-7.

Zhang H, Zhu Y-J, Fan Y-Y, Huang T-X, Zhang J-F, Xie H-A, Zhuang J-Y. 2019. Identification and verification of quantitative trait loci for eating and cooking quality of rice (Oryza sativa). Plant Breeding 138: 568-576 DOI 10.1111/pbr.12704.

Peer) reviewing PDF | (2019:11:43048:1:0:NEW 10 Jan 2020) 
439

440

441

442

443

444

445

446

447

448

449

450

451

452

453

454

455

456
Zhang H-W, Fan Y-Y, Zhu Y-J, Chen J-Y, Yu S-B, Zhuang J-Y. 2016. Dissection of the qTGW1.1 region into two tightly-linked minor QTLs having stable effects for grain weight in rice. BMC Genet 17: 98 DOI 10.1186/s12863-016-0410-5.

\section{Zhang X, Wang J, Huang J, Lan H, Wang C, Yin C, Wu Y, Tang H, Qian Q, Li J, Zhang H.}

2012. Rare allele of OsPPKL1 associated with grain length causes extra-large grain and a significant yield increase in rice. Proceedings of the National Academy of Sciences of the United States of America 109: 21534-21539 DOI 10.1073/pnas.1219776110.

Zhao D-S, Li Q-F, Zhang C-Q, Zhang C, Yang Q-Q, Pan L-X, Ren X-Y, Lu J, Gu M-H, Liu Q-Q. 2018. GS9 acts as a transcriptional activator to regulate rice grain shape and appearance quality. Nature Communication 9: 1240 DOI 10.1038/s41467-018-03616-y.

Zheng K, Huang N, Bennett J, Khush GS. 1995. IRRI Discussion Paper Series No.12. Manila: International Rice Research Institute.

Zhu Y-J, Sun Z-C, Niu X-J, Ying J-Z, Fan Y-Y, Mou T-M, Tang S-Q, Zhuang J-Y. 2019a. Dissection of three quantitative trait loci for grain size on the long arm of chromosome 10 in rice (Oryza sativa L.). Peer J 7: e6966 DOI 10.7717/peerj.6966.

Zhu Y, Zhang Z, Chen J, Fan Y, Mou T, Tang S, Zhuang J. 2019b. Fine mapping of qTGW10-20.8, a QTL having important contribution to grain weight variation in rice The Crop Journal 7: 587-597 DOI 10.1016/j.cj.2019.01.006. 


\section{Figure 1}

Figure 1 Development of nine populations used in this study.

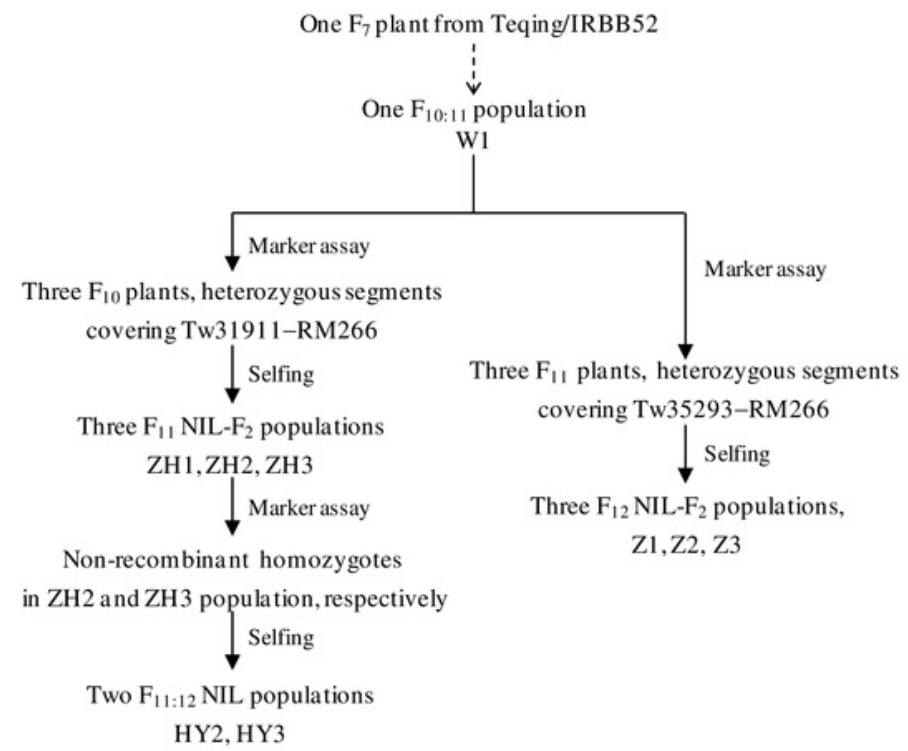


Figure 2

Figure 2 Genomic distribution of QTLs for four traits detected in the W1 population.

TGW=1000-grain weight; GL=Grain length; GW=Grain width; LWR=Length/width ratio. The markers flanking GS2, GS3, and GW5 are indicated in boldface. 

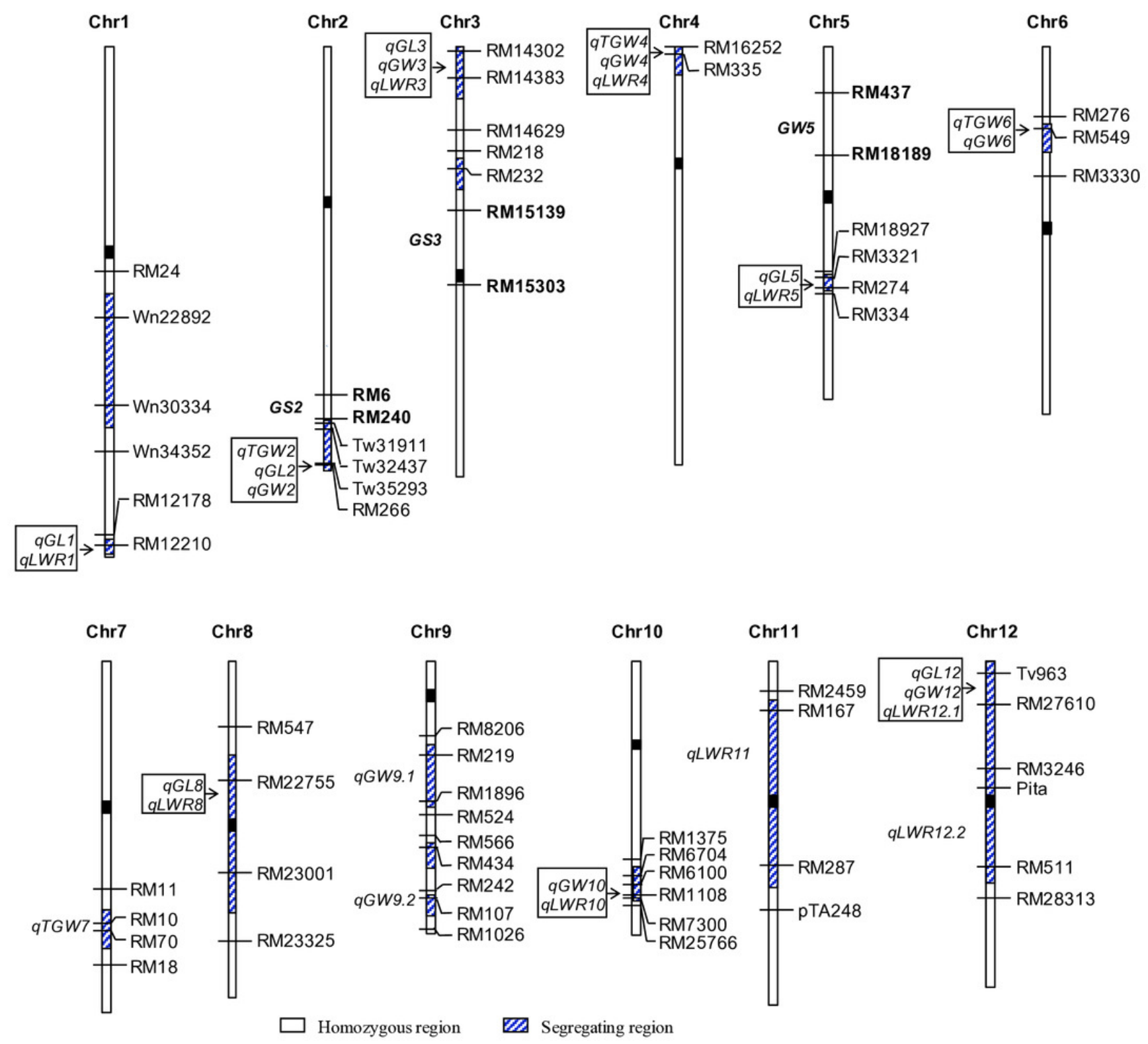


\section{Figure 3}

Figure 3 Segregating regions of the eight populations.

(A) Three populations in $\mathrm{F}_{11}$; (B) Segregating regions of the three populations in $\mathrm{F}_{11}$ were updated with new polymorphic markers. Two sets of NIL populations in $\mathrm{F}_{11: 12}$ were used to validate the genetic effects of qTGW2; (C) Three populations in $\mathrm{F}_{12}$ were used for finemapping of $q T G W 2$. 
A

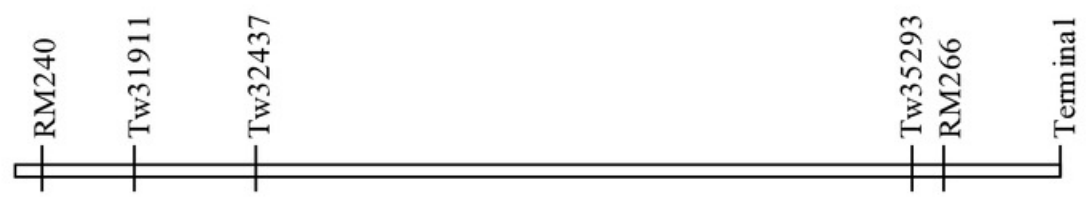

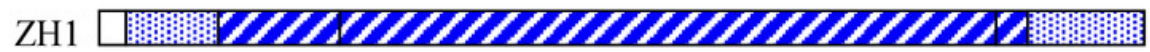

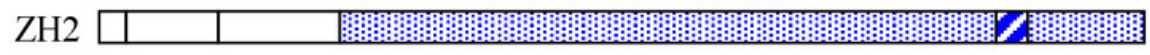
ZH3 $\mathrm{Z}$ -

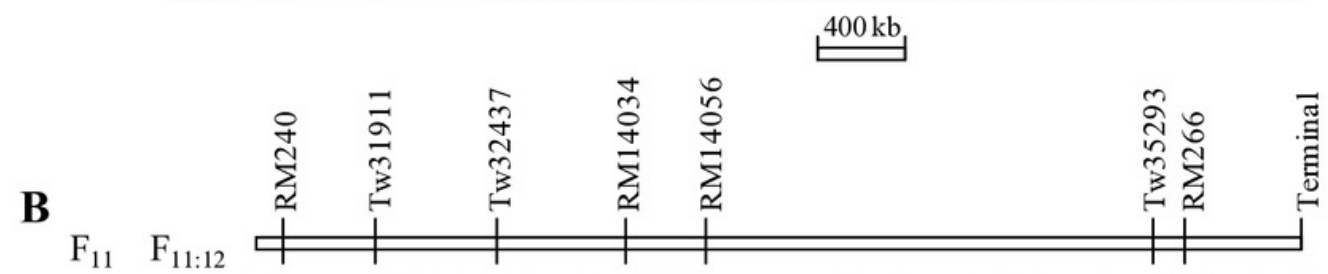

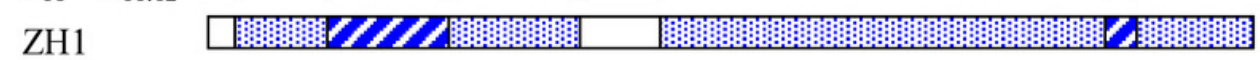

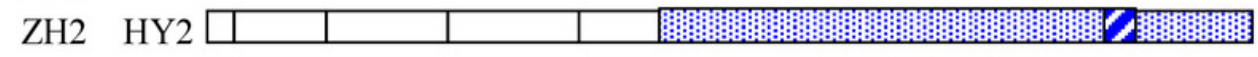
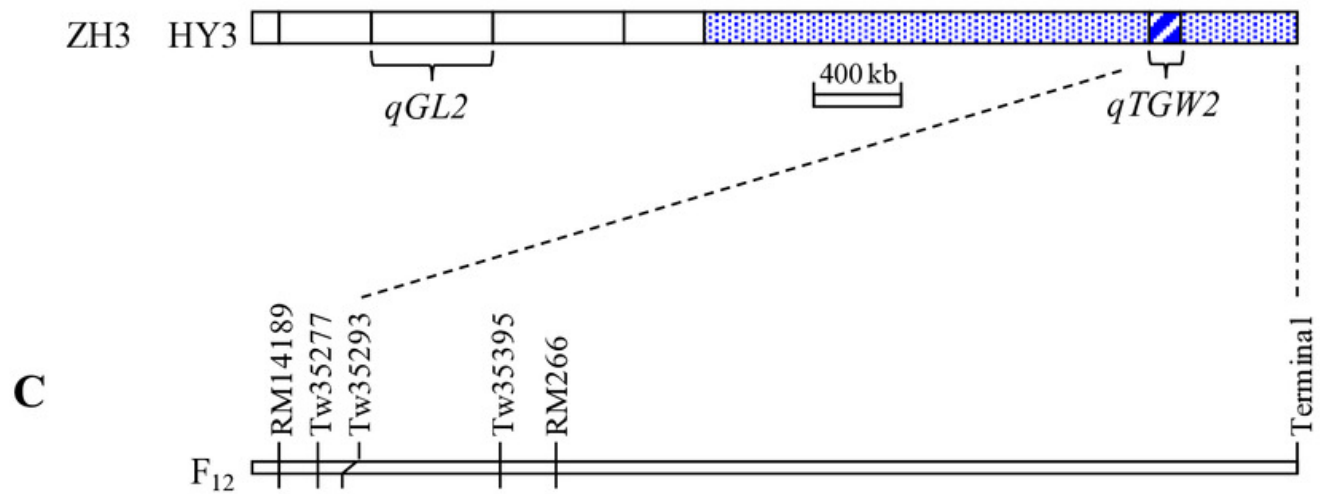

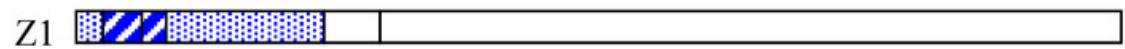

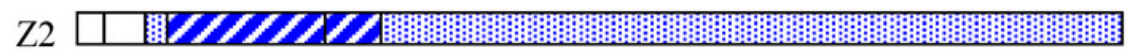

Z3 ㄴ $\underset{q T G W 2}{60 \mathrm{~kb}}$

$\square$ Homozygous region $\mathbb{Z}$ Segregating region $\quad$ 眮 Crossover region 
Figure 4

Figure 4 Distributions of 1000-grain weight, grain length and width, length/width ratio in $\mathrm{HY} 2$ and HY3.

$\mathrm{NIL}^{\mathrm{TQ}}$ and NIL ${ }^{\text {IRBB52 }}$ are near isogenic lines (NILs) having Teqing and IRBB52 homozygous genotypes in the segregating region, respectively.

HY2
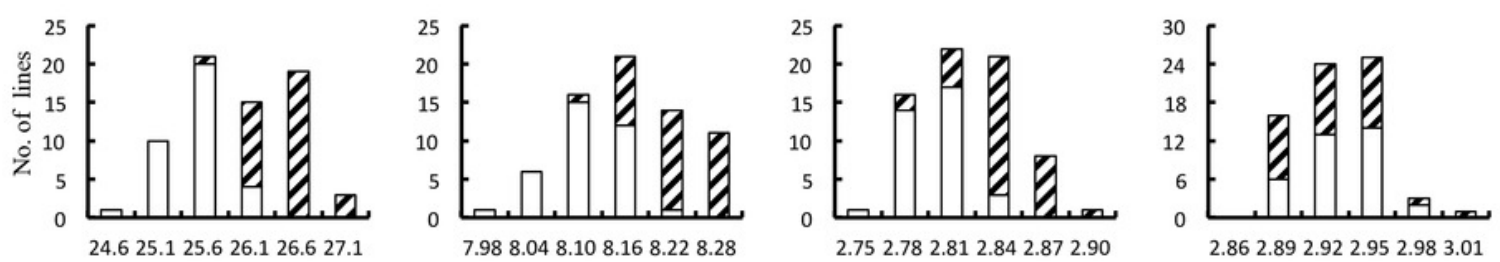
HY3
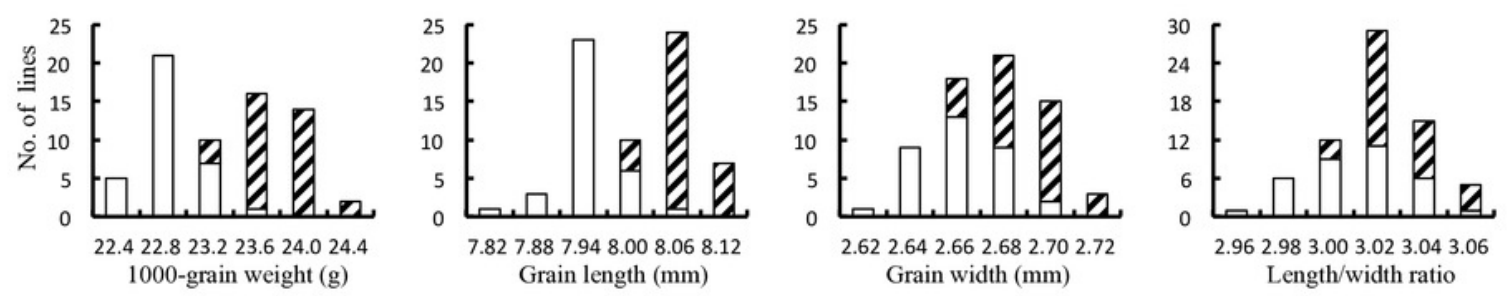

$$
\square \mathrm{NIL}^{\mathrm{TQ}} \square \mathrm{NIL}^{\mathrm{IRBB} 52}
$$




\section{Table 1 (on next page)}

Table 1 QTLs detection for four traits using the W1 population. 
Table 1 QTLs detection for four traits using the W1 population

\begin{tabular}{|c|c|c|c|c|c|c|}
\hline Chr. & Interval & QTL & $L O D$ & $A$ & $D$ & $R^{2}(\%)$ \\
\hline \multirow[t]{2}{*}{1} & RM12210 & $q G L 1$ & 3.82 & 0.026 & -0.017 & 3.96 \\
\hline & & $q L W R 1$ & 5.09 & 0.011 & -0.013 & 3.30 \\
\hline \multirow[t]{3}{*}{2} & Tw35293-RM266 & $q T G W 2$ & 11.76 & 0.39 & 0.15 & 14.50 \\
\hline & & $q G L 2$ & 15.49 & 0.060 & 0.006 & 18.60 \\
\hline & & $q G W 2$ & 6.15 & 0.013 & 0.006 & 5.69 \\
\hline \multirow[t]{3}{*}{3} & RM14302-RM14383 & $q G L 3$ & 3.81 & 0.025 & 0.027 & 4.01 \\
\hline & & $q G W 3$ & 7.26 & -0.014 & -0.004 & 6.91 \\
\hline & & $q L W R 3$ & 14.99 & 0.022 & 0.005 & 11.24 \\
\hline \multirow[t]{3}{*}{4} & RM16252-RM335 & qTGW4 & 3.01 & -0.18 & 0.06 & 3.41 \\
\hline & & $q G W 4$ & 11.81 & -0.017 & -0.013 & 11.21 \\
\hline & & $q L W R 4$ & 15.26 & 0.021 & 0.020 & 11.67 \\
\hline \multirow[t]{2}{*}{5} & RM3321-RM274 & $q G L 5$ & 17.79 & 0.062 & 0.005 & 21.67 \\
\hline & & $q L W R 5$ & 11.31 & 0.018 & -0.009 & 8.39 \\
\hline \multirow[t]{2}{*}{6} & RM549 & qTGW6 & 6.58 & 0.27 & -0.22 & 7.41 \\
\hline & & $q G W 6$ & 5.82 & 0.012 & -0.004 & 5.19 \\
\hline 7 & RM10-RM70 & $q T G W 7$ & 12.15 & 0.39 & -0.09 & 15.27 \\
\hline \multirow[t]{2}{*}{8} & RM22755-RM23001 & $q G W 8$ & 3.48 & -0.009 & -0.010 & 3.20 \\
\hline & & $q L W R 8$ & 9.84 & 0.017 & 0.005 & 7.08 \\
\hline \multirow[t]{2}{*}{9} & RM219-RM1896 & $q G W 9.1$ & 3.70 & 0.009 & 0.012 & 3.88 \\
\hline & RM107 & $q G W 9.2$ & 7.13 & 0.012 & 0.010 & 6.39 \\
\hline \multirow[t]{2}{*}{10} & RM1108-RM7300 & $q G W 10$ & 4.45 & -0.004 & -0.029 & 4.34 \\
\hline & & $q L W R 10$ & 8.00 & 0.012 & 0.023 & 5.28 \\
\hline 11 & RM167-RM287 & $q L W R 11$ & 7.58 & -0.016 & -0.019 & 7.05 \\
\hline \multirow[t]{3}{*}{12} & Tv963-RM27610 & qGL12 & 3.85 & 0.031 & 0.015 & 4.95 \\
\hline & & $q G W 12$ & 7.00 & -0.013 & -0.010 & 6.24 \\
\hline & & $q L W R 12.1$ & 23.37 & 0.027 & 0.013 & 18.05 \\
\hline 12 & Pita-RM511 & $q L W R 12.2$ & 4.59 & -0.010 & 0.015 & 3.22 \\
\hline
\end{tabular}

Notes.

TGW = 1000-grain weight (g); GL = Grain length (mm); GW = Grain width (mm); LWR = Length/width ratio; $A$ : additive effect of replacing a Teqing allele with a IRBB52 allele; $D$ : dominance effect; $R^{2}$ : proportion of the phenotypic variance explained by the QTL. 
Table 2 (on next page)

Table 2 Dissection of two QTLs using three NIL-F $F_{2}$ populations. 
Table 2 Dissection of two QTLs using three NIL-F 2 populations

\begin{tabular}{lllrrrr}
\hline Population & Segregating region & Trait & LOD & \multicolumn{1}{c}{$A$} & \multicolumn{1}{c}{$D$} & $R^{2}(\%)$ \\
\hline ZH1 & Tw31911-Tw32437 & TGW & 2.83 & -0.20 & -0.15 & 6.05 \\
& & GL & 12.06 & -0.069 & -0.049 & 29.51 \\
& & GW & 5.77 & 0.004 & -0.024 & 13.61 \\
& & LWR & 5.30 & 0.004 & 0.050 & 28.52 \\
& \multirow{2}{*}{ Tw35293-RM266 } & TGW & 14.79 & 0.46 & -0.19 & 31.76 \\
& & GL & 15.54 & 0.056 & -0.008 & 17.95 \\
& & GW & 1.87 & 0.006 & -0.003 & 3.73 \\
& & LWR & 0.17 & & & \\
ZH2 & Tw35293-RM266 & TGW & 17.60 & 0.55 & -0.02 & 28.63 \\
& & GL & 13.15 & 0.065 & -0.022 & 22.34 \\
& & GW & 13.54 & 0.021 & -0.001 & 22.91 \\
& & LWR & 0.39 & & & \\
ZH3 & Tw35293-RM266 & TGW & 12.52 & 0.46 & 0.03 & 21.83 \\
& & GL & 17.42 & 0.061 & -0.006 & 28.86 \\
& & GW & 12.81 & 0.020 & 0.001 & 22.37 \\
& & LWR & 0.38 & & & \\
\hline
\end{tabular}

\section{Notes.}

TGW = 1000-grain weight (g); GL = Grain length (mm); GW = Grain width (mm); LWR = Length/width ratio; $A$ : additive effect of replacing a Teqing allele with a IRBB52 allele; $D$ : dominance effect; $R^{2}$ : proportion of the phenotypic variance explained by the QTL. 


\section{Table 3(on next page)}

Table 3 Validation of qTGW2 using two NIL populations. 
Table 3 Validation of $q$ TGW2 using two NIL populations

\begin{tabular}{lcllrrr}
\hline Population & Trait & \multicolumn{2}{l}{ Phenotype ( Mean \pm SD $)$} & $P$ & $A$ & $R^{2}(\%)$ \\
\cline { 2 - 4 } & & \multicolumn{2}{l}{ NIL $^{\text {TQ }}$} & & & \\
\hline HY2 & TGW & $25.23 \pm 0.29$ & $26.17 \pm 0.34$ & $<0.0001$ & 0.47 & 60.94 \\
& GL & $8.081 \pm 0.045$ & $8.189 \pm 0.049$ & $<0.0001$ & 0.054 & 48.26 \\
& GW & $2.784 \pm 0.021$ & $2.826 \pm 0.025$ & $<0.0001$ & 0.021 & 36.67 \\
& LWR & $2.915 \pm 0.024$ & $2.911 \pm 0.031$ & 0.5690 & & \\
HY3 & TGW & $22.65 \pm 0.24$ & $23.56 \pm 0.26$ & $<0.0001$ & 0.45 & 66.02 \\
& GL & $7.919 \pm 0.041$ & $8.040 \pm 0.036$ & $<0.0001$ & 0.061 & 62.12 \\
& GW & $2.652 \pm 0.017$ & $2.678 \pm 0.018$ & $<0.0001$ & 0.013 & 22.74 \\
& LWR & $3.000 \pm 0.022$ & $3.017 \pm 0.015$ & 0.0003 & 0.009 & 12.94 \\
\hline
\end{tabular}

Notes.

TGW = 1000-grain weight (g); GL = Grain length (mm); GW = Grain width (mm); LWR = Length/width ratio; A: additive effect of replacing a Teqing allele with a IRBB52 allele; D: dominance effect; R2: proportion of the phenotypic variance explained by the QTL. NIL ${ }^{\text {TQ }}$

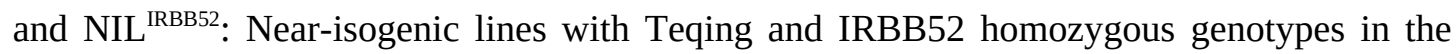
segregating region, respectively. 
Table 4 (on next page)

Table 4 Fine mapping of $q T$ TGW2 using three NIL- $F_{2}$ populations. 
Manuscript to be reviewed

Table 4 Fine mapping of $q T G W 2$ using three NIL-F 2 populations

\begin{tabular}{|c|c|c|c|c|c|c|c|c|}
\hline \multirow[t]{2}{*}{ Population } & \multirow[t]{2}{*}{ Trait } & \multicolumn{3}{|c|}{ Phenotype ( Mean \pm SD } & \multirow[t]{2}{*}{$L O D$} & \multirow[t]{2}{*}{$A$} & \multirow[t]{2}{*}{$D$} & \multirow[t]{2}{*}{$R^{2}(\%)$} \\
\hline & & Teqing & IRBB52 & Heterozygote & & & & \\
\hline \multirow[t]{4}{*}{ Z1 } & TGW & $24.03 \pm 0.53$ & $23.99 \pm 0.51$ & $23.94 \pm 0.66$ & 0.20 & & & \\
\hline & GL & $8.017 \pm 0.073$ & $8.004 \pm 0.072$ & $8.004 \pm 0.065$ & 0.26 & & & \\
\hline & GW & $2.805 \pm 0.032$ & $2.809 \pm 0.036$ & $2.808 \pm 0.034$ & 0.06 & & & \\
\hline & LWR & $2.858 \pm 0.026$ & $2.850 \pm 0.026$ & $2.851 \pm 0.025$ & 0.65 & & & \\
\hline \multirow[t]{4}{*}{$\mathrm{Z} 2$} & TGW & $24.96 \pm 0.54$ & $25.90 \pm 0.51$ & $25.33 \pm 0.49$ & 18.38 & 0.46 & -0.10 & 29.93 \\
\hline & GL & $8.156 \pm 0.094$ & $8.266 \pm 0.091$ & $8.205 \pm 0.087$ & 9.30 & 0.053 & -0.008 & 16.26 \\
\hline & GW & $2.976 \pm 0.026$ & $3.004 \pm 0.029$ & $2.986 \pm 0.022$ & 7.76 & 0.014 & -0.004 & 13.70 \\
\hline & LWR & $2.750 \pm 0.041$ & $2.762 \pm 0.037$ & $2.757 \pm 0.031$ & 0.68 & & & \\
\hline \multirow[t]{4}{*}{$\mathrm{Z3}$} & TGW & $26.26 \pm 0.88$ & $26.34 \pm 0.73$ & $26.20 \pm 0.84$ & 0.22 & & & \\
\hline & GL & $8.272 \pm 0.111$ & $8.262 \pm 0.078$ & $8.271 \pm 0.113$ & 0.07 & & & \\
\hline & GW & $3.046 \pm 0.046$ & $3.054 \pm 0.027$ & $3.043 \pm 0.046$ & 0.51 & & & \\
\hline & LWR & $2.726 \pm 0.070$ & $2.714 \pm 0.029$ & $2.727 \pm 0.067$ & 0.39 & & & \\
\hline
\end{tabular}

Notes.

TGW = 1000-grain weight (g); GL = Grain length (mm); GW = Grain width (mm); LWR = Length/width ratio; $A$ : additive effect of replacing a Teqing allele with a IRBB52 allele; $D$ : dominance effect; $R^{2}$ : proportion of the phenotypic variance explained by the QTL. 